\title{
Les catégories d'âge face aux mutations temporelles de la société
}

Marc Bessin ${ }^{1}$

Article pour la revue Gérontologie et société, N77, "Le temps", décembre 1996.

"Désolé, vous n'avez plus l'âge !" "Vous êtes trop jeune pour y avoir droit !" Symbole de la rationalité administrative, les critères d'âge, exprimés de façon rigide par l'état civil, sont tous les jours utilisés par les institutions pour convoquer des administrés, ouvrir des droits ou supprimer des allocations ${ }^{2}$. Cette pression de l'âge chronologique dans la vie quotidienne, dont on peut voir un dernier exemple dans la proposition d'inéligibilité des personnes âgées de plus de 75 ans, est pourtant contestée, et certaines institutions l'abandonnent même au bénéfice de critères plus individualisés, liés aux situations de chacun.

S'interroger sur les seuils d'âge revient à analyser l'activité ordinaire qui consiste à construire des catégories et à classifier la population en fonction du temps biographique. Cet article propose de déconstruire cette opération de catégorisation par âges, en considérant qu'au centre de l'ambivalence vis-à-vis de l'âge dans la façon d'intervenir sur les itinéraires biographiques des personnes, s'exprime l'avènement difficile d'une mutation profonde de la société en matière de régulation temporelle.

Les mutations temporelles de la société incitent à renouveler les modes de régulation biographique, mais n'amènent-elles pas surtout à réinterroger les catégories pour penser les temps de la vie?

\section{Le Chronos : réduire la vie en un temps unique}

La façon d'organiser, de rythmer et de ritualiser les différents segments temporels est un trait fondamental de la culture d'une société. Les sociétés industrielles, produit d'un long processus de rationalisation, ont en particulier forgé une représentation du temps fortement ancrée, celle de l'Horloge, qui réduit les différents registres de la vie des individus en un temps unique.

\section{La culture temporelle industrielle}

La multiplication des échanges, le développement des transports et la complexité croissante de la société ont engendré un besoin de plus en plus croissant d'un temps commun standardisé pour assurer la coopération sociale et le fonctionnement de l'économie capitaliste. Le système GMT symbolise cette standardisation du temps (Zerubavel, 1982) qui est au coeur de la construction du monde moderne (Landes, 1983). C'est le temps du Chronos qui s'impose lentement comme la représentation temporelle dominante, rompant avec une conception où se sont les pratiques sociales qui rythmaient la vie sociale sans forcément obéir à des principes de programmation et de prévision (Bourdieu, 1963). Cette rationalisation du temps conduit à différencier l'activité humaine du cadre temporel préalable et abstrait dans lequel elle s'effectue. Linéaire et segmenté, le temps de cette culture industrielle est marqué par la monochronie (Hall, 1983) qui cloisonne les activités en attribuant une seule fonction à chaque segment temporel. Ainsi le temps du travail salarié est fortement distinct des autres temps sociaux. Il est dominant dans ce modèle temporel,

${ }^{1}$ ) Sociologue, chargé de recherche au CNRS, Groupe de Recherche sur la Socialisation, Université Lumière Lyon II, 5 av. P.Mendès-France, 69676 Bron cedex.

${ }^{2}$ ) Une partie de cet article reprend une version largement remaniée d'un texte paru dans la revue Temporalistes (Bessin, 1994). 
non seulement parce qu'il est quantitativement central, sur tous les segments temporels (journée, année, durée de l'existence), mais surtout parce qu'il structure tous les temps sociaux qui s'organisent en référence à ce temps pivot (Sue, 1994). Par sa rationnalité, ce temps de la modernité est orienté vers un futur à construire, en ce sens il est cumulatif et irréversible.

\section{L'institution biographique}

Les éléments culturels de ce temps industriel se sont traduits, au niveau des trajectoires individuelles, en normes d'âges, limites et seuils d'âge, agents spécialisés, institutions, etc. : le cours de vie s'est institutionnalisé.

Une organisation ternaire des trajectoires biographiques, segmentées en différents âges de la vie et scandées par le passage successif dans les institutions de socialisation, s'est imposée dans l'après-guerre. L'âge adulte, défini essentiellement par l'intégration dans une famille et dans un emploi, constitue le pivot de ce modèle du cours de vie, autour duquel se polarisent la jeunesse caractérisée par le fait d'être en formation et la vieillesse en retraite. La domination du temps de travail dans la structure temporelle s'illustre dans la façon dont les différents registres de la vie se synchronisent en fonction des calendriers professionnels de l'homme, eu égard à la division sexuelle du travail. Ainsi, le mariage, célébré à un âge de plus en plus jeune de 1935 à 1970, intervenait après une entrée définitive dans le travail, et très rapidement la naissance d'enfants s'ensuivait. Le modèle de "l'emploi à vie" de la société salariale (Castel, 1995) permettait d'envisager des carrières professionnelles stables, linéaires et ascendantes. Le monisme familial qui caractérisait cette époque sur le modèle de la famille nucléaire permettait de définir un calendrier familial relativement prévisible pour une immense majorité des personnes. La fonction de scansion temporelle des seuils d'âge ne joue que dans la mesure où ils rythment les biographies en affectant une place stable aux individus dans la société de façon durable. C'est le temps de la certitude. Cette apparente stabilité laissait peu de place aux réversibilités dans les biographies.

Scander, segmenter, synchroniser et spécialiser, tels peuvent être résumés les procédés de l'institutionnalisation du cours de vie, qui repose sur l'âge chronologique comme critère de classement et de mise en ordre "naturel" de la société. Cette chronologisation du cours de vie illustre en fait le triomphe de Chronos : un temps quantitatif et linéaire, dominé par la ponctualité et l'exactitude, qui a abouti à cette sorte de lit de Procuste de la temporalité qui nous paraît parfois si rigide.

\section{La chronologisation du cours de vie}

Le procédé qui consiste à rapporter l'organisation du cours de vie sur une échelle quantitative du temps graduée en âges semble tomber sous le sens. Il faut pourtant situer historiquement l'utilisation de cette "variable objective" en la rapportant à l'avènement d'un temps quantifiable. Les âges exacts sont restés longtemps peu connus par les individus eux-mêmes. La généralisation de l'inscription de la naissance sur les registres paroissiaux s'est effectuée au seizième siècle en France, faisant ainsi apparaître l'âge d'état civil comme critère de classement. C'est avec la Modernité que l'âge chronologique est devenu petit à petit un critère systématiquement appliqué pour classer, catégoriser, autoriser ou prohiber tel ou tel droit ou accès. Cette utilisation institutionnalisée a engendré une plus grande distinction des "âges de la vie" en mettant en relation l'identité à l'âge. En ce sens, l'âge est à la fois le produit et l'instrument des institutions. Cette "police des âges", qui a touché tous les domaines, répond à un souci d'intégration et de synchronisation sociales par une standardisation des existences. Les propriétés fondamentales de l'âge - neutralité apparente et simplicité d'utilisation - nous aident à en mieux comprendre le 
recours systématique.

\section{L'âge chronologique, un critère "démocratique" et rationnel}

Le critère de l'âge de l'état civil semble tout ce qu'il y a de plus neutre et objectif. Ses propriétés rationnelles en font pour le statisticien une variable parfaite et rêvée (Thévenot, 1979) et pour qui veut réglementer ou classer, un instrument de gestion extraordinairement pratique. Pouvant s'appliquer à chacun sans trop de contestation, il est accepté par tous. En ce sens, parce que l'âge chronologique met l'ensemble des individus égaux entre eux devant les différentes institutions qui régissent selon ce critère, l'utilisation de ce procédé s'est trouvée naturellement justifiée. Les "garanties" de neutralité et d'égalité offertes par la rationalité et l'objectivité sont au fondement de l'intervention publique depuis la Révolution Française. C'est ainsi qu'au nom de la démocratie, l'âge chronologique est devenu le principe régulateur majeur de la population dans toutes les institutions (Percheron, 1991).

Si l'école, centrale dans le processus d'institutionnalisation du cours de vie (Chamboredon, 1991), offre un bon exemple d'utilisation rigide des critères d'âge, où ils fonctionnent comme outil de sélection, ceux-ci se sont surtout imposés avec l'Etat-providence. Les institutions destinées à faire face aux aléas de la vie des individus, sur le principe de la solidarité et de l'assurance, s'appuient sur un modèle de parcours biographique en ciblant des catégories de population clairement définies, notamment en fonction de l'âge. La gestion des carrières professionnelles et des retraites a ainsi vu progressivement s'effacer la notion de durée devant celle de seuil d'âge, faisant de l'âge fixe et rigide de la retraite un véritable couperet pour des travailleurs s'étant constamment identifié à leur place dans le travail.

\section{L'intériorisation des normes d'âge}

La généralisation et la banalisation de l'utilisation du critère d'âge ont fait de l'écart à la norme d'âge une donnée essentielle dans la représentation sociale. Aussi l'institutionnalisation du cours de vie consiste autant à cloîtrer les individus dans des âges sociaux définis par leur âge chronologique, que de permettre aux personnes de s'adapter à cet enfermement.

Bernice Neugarten, en développant l'idée d'une horloge interne aux individus, a par exemple montré qu'ils ont conscience de ce calendrier et qu'ils se positionnent en fonction de ces dates considérées comme normales. Le processus de normalisation des âges de la vie n'est pas seulement fait de règles formelles, mais constitue une carte cognitive en chacun d'entre nous. Cette carte mentale du cours de vie permettrait de prévoir certains événements à venir, et d'anticiper de cette manière des situations où l'on est ou non conforme au temps, afin de ne pas complètement les subir (Neugarten \& Hagestad, 1976).

L'intériorisation des normes d'âge conjugue donc des éléments relevant de la Loi (les majorités, les limites d'âge...) et d'autres relevant du contrôle social qui "comprennent, bien que leur action et leurs effets soient plus insidieux, les dispositifs cognitifs, symboliques et rituels qui entraînent l'adhésion de l'individu (...). C'est par eux que l'ordre social est mis en analogie avec celui de la nature, donnant à croire qu'il existe une nature sociale à laquelle on ne commande qu'en lui obéissant." (Balandier, 1985). On peut illustrer ces deux domaines par la régulation sociale de l'âge de la maternité, à laquelle contribue par exemple la fixation d'un âge à partir duquel est prise en charge l'amniocentèse ${ }^{1}$. Mais la limitation sociale du temps de fertilité des femmes (beaucoup plus contraignante que les limites physiologiques du corps humain) n'est efficace que si la stigmatisation des maternités tardives, par le monde médical et l'environnement professionnel et familial, relaie les mesures législatives ou administratives (Valabregue \& al.,

\footnotetext{
1) Actuellement fixé à 38 ans, le gouvernement s'oppose actuellement "pour des raisons budgétaires" à étendre le dépistage anténatal de la trisomie 21 aux plus jeunes.
} 
1982).

D'ordre institutionnel et culturel (de Conninck \& Godard, 1990), la normalisation des âges de la vie selon une chronologie assez précise traduit, au niveau des temporalités individuelles, le mode de régulation temporelle dominant dans les sociétés industrielles. Le Chronos reposait sur la synchronisation des différents calendriers biographiques correspondant au cloisonnement des registres de la vie structurés autour d'un temps pivot de référence. Les mutations actuelles tendent à remettre en cause cette idée de structure temporelle de la société. C'est l'examen de cet éclatement des temporalités et de la façon dont il se répercute sur le cours de vie que nous examinerons.

\section{L'éclatement des temporalités}

Les mutations sociales qui ont particulièrement affecté les sphères de la famille et du travail salarié depuis les années 70 peuvent être lues comme la remise en cause de la culture temporelle industrielle, avec la montée d'une flexibilité temporelle marquée par l'incertitude, l'instabilité, la dilution des repères et l'arasement des seuils.

\section{La problématique familiale assume le temps}

Alors que l'édifice de la famille moderne s'est laborieusement construit autour du modèle unique de la famille nucléaire, le pluralisme familial a mis moins de vingt-cinq ans à s'imposer. Les nouveaux régimes familiaux, issus des recompositions des formes que prend une famille au cours de son existence, sont générateurs de nouvelles perceptions du temps. Plus précisèment, la problématique familiale actuelle revient à intégrer le temps non plus comme une menace, mais comme un contexte, en considérant la temporalité d'une vie vécue non plus comme un état, mais comme un processus (Théry, 1996). La crise du mariage symbolise une crise profonde des engagements durables en matière d'union. L'incertitude domine les temporalités familiales de l'alliance, dès lors la problématique familiale se recentre sur le lien de filiation. Le bouleversement des temporalités des phénomènes démographiques tient d'une individualisation des choix et d'une plus grande liberté de programmation, en matière de fertilité notamment, dans un contexte où les femmes doivent majoritairement gérer une double carrière, familiale et professionnelle. Les calendriers familiaux deviennent ainsi plus réversibles et se diversifient : mieux maîtrisés individuellement, ils sont moins prévisibles socialement.

\section{Les dérégulations de l'emploi}

De son côté, le travail salarié ne constitue plus un donneur de temps pour l'ensemble de la société. La diminution quantitative de la durée du travail au niveau de tous les segments temporels, la place de plus en plus importante allouée à d'autres temps sociaux et notamment au temps libre, la part de la formation dans les carrières et la montée de l'exclusion par le chômage contribuent à une dérégulation du travail salarié. La mobilité que nécessite la participation à la compétition du travail, ou la précarité subie pour ceux qui en sont écartés, participent à leur niveau à l'idée de flexibilité du travail. Les figures de l'exclusion et de la précarité produites par ces évolutions ne sont que le résultat d'une onde de choc qui ébranle toute la société salariale (Castel, 1995), exprimant des processus plus fondamentaux de fragilisation de l'inscription sociale. La flexibilité temporelle s'avère ainsi le résultat conjugué d'un desserrement des contraintes et d'une précarisation des statuts. Il en résulte une moindre prégnance des normes temporelles due à l'incapacité des institutions d'imposer des normes univoques à tous les individus. 


\section{L'émergence du Kairos}

L'évolution technologique n'inspire plus la confiance que leur octroyait l'idéologie du progrès : la montée des préoccupations éthiques et écologiques révèle la "crise de l'avenir" (Pomian, 1980). Les difficultés de l'Etat-providence à protéger des risques sociaux et de la désaffiliation (Castel, 1995) confirment un retour de l'imprévisibilité et une impossibilité d'appréhender un futur programmé. L'incertitude prévaut dans les sphères de la famille comme de l'emploi, et si notre société se tourne parfois sur son passé, elle vit surtout dans un rapport intense avec le présent. Les techniques visant à supprimer le temps (communication en temps réel, flux tendus, etc.) ne font que renforcer ce "présentisme" (Hartog, 1995) qui rompt avec le régime moderne d'historicité auxquels se rattachaient l'avenir, le progrés et l'idéologie.

Alors que l'affirmation d'un Temps unique caractérisait la représentation sociale, c'est la diversité des temps sociaux, leur enchevêtrement et la pluralité de leur mode d'organisation qui semblent maintenant à l'ordre du jour. Cette dérégulation entraîne un sentiment de crise anomique dans la mesure où les repères temporels se diluent. Les catégories de temps, construites à partir de divisions engendrées par le cloisonnement et la linéarité, auxquelles on attribue des fonctions (temps de travail, temps de formation, loisirs, etc.), se brouillent en raison de leurs enchâssements perpétuels et de l'impossibilité de dégager un temps social dominant. Les mutations temporelles peuvent ainsi être interprétées comme une impasse de la modernité dans sa tentative de réduire les différents temps sociaux dans le seul registre du Chronos.

Avec la prégnance de l'imprévisible et de l'instabilité, une autre dimension du temps apparaît, celle du Kairos, rappelant que "la première logique du vivant consiste à créer le temps de son existence" (Grossin, 1996, p.18). Le Kairos suggère l'intempestif, l'opportunité, le moment favorable ou l'occasion propice. On est ici dans le domaine de l'action pratique et du temps de l'action en train de se faire. Une dimension plus qualitative du temps est présente dans cette dimension, qui assume d'emblée un éventail de temps (la polychronie), le problème étant justement de choisir dans cette diversité le moment adapté à la situation. Le Kairos renvoie en effet à une figure du temps beaucoup moins rationalisée et définie à l'avance, dépendant des situations. En ce sens, il s'agit d'une dimension plus pragmatique du temps, qui relève du sens pratique (Bessin, 1997).

\section{Ages et parcours de vie face aux dérégulations}

Enfermés dans une rationalité liée à la flèche du temps linéaire, nous avons du mal à appréhender ce registre de l'expérience temporelle (Kairos), qui semble en tout cas difficilement compatible avec la tradition moderne de l'intervention des institutions participant à la régulation des temporalités biographiques. C'est en commençant par analyser un secteur au coeur des processus de dérégulation, les politiques de l'emploi et la façon dont les critères d'âge y ont été utilisés, que nous examinerons des changements de l'institution biographique liés à ces mutations temporelles.

\section{L'âge dans les politiques d'emploi en période de crise}

Comment le critère d'âge chronologique a-t-il été utilisé dans la régulation du marché de travail ? Cette question renvoie directement aux contradictions des politiques d'emploi en période de crise. L'âge est en effet apparu fortement discriminant dans ce domaine : un véritable outil de gestion du chômage. Les mesures d'âge ont été les premières réponses à la crise naissante de l'emploi. A partir de 1973, la généralisation de l'indemnisation des chômeurs vieillissants s'est rapidement transformée en une exclusion des travailleurs âgés à travers l'explosion des préretraites. Parallèlement, l'entrée dans l'emploi s'est vue bloquée pour les jeunes. Une sorte de compromis 
s'est en fait établie entre les syndicats, le patronat et l'Etat pour maintenir dans un premier temps un noyau stable du salariat protégé en sacrifiant les plus âgés et les plus jeunes (Gaullier, 1988).

\section{La gestion des fins de carrières professionnelles}

Pour les salariés âgés, on a vu apparaître dans la deuxième moitié des années 70 un phénomène massif d'arrêt prématuré de la carrière professionnelle, vécu comme un couperet brutal et comme une exclusion du monde social encore plus douloureuse que ne l'était la retraite, sur fond de restructurations industrielles. En ce sens, les préretraites ont d'abord amplifié une spécialisation et une ségrégation des âges. Toutefois, l'évolution de la fin d'activité ne peut se résumer à un seul changement de calendrier. Dans les années 80, le chômage structurel s'est installé en atteignant aujourd'hui tous les âges. Si les préretraites continuent encore à faire office de "politique de l'emploi" (bel euphémisme !), ce sont surtout de "nouvelles formes de transition entre activité et retraite" (Guillemard, 1993) qui se sont imposées, liées à un recul des systèmes de retraite comme instruments centraux de régulation des sorties d'activité. On aboutit à un reflux des critères d'âge chronologique au profit de critères fonctionnels. La notion d'âge de la retraite n'a plus de sens car ce seuil d'âge est arasé par la flexibilité des fins de carrières professionnelles qui se transforment par la multiplication de statuts intermédiaires évolutifs entre l'activité et la retraite. La "déchronologisation" de ce passage marque la fin d'un modèle standardisé qui faisait de cette transition un repère commun à tous.

Il est important de noter que cette flexibilisation des fins de carrières est caractérisée par un déficit d'autonomie individuelle. Nous sommes encore loin d'un passage progressif et volontaire à la retraite pour tous et d'une participation active des retraités à la vie de la cité.

\section{Les politiques d'insertion professionnelle}

Pour l'entrée en activité, les dispositifs d'insertion professionnelle ont également pris des distances avec les critères d'âge stricts. Destinés au départ à un public restreint au niveau de l'âge, ils se sont au fur et à mesure étendus pour dans certaines occasions atteindre tous les âges. Ce fut notamment le cas d'opérations telles que les "Nouvelles Qualifications" ou de dispositifs comme les "contrats de formation individualisée"(CFI)1, expérimentés auprès des jeunes et généralisés ou étendus aux adultes. Pour résumer rapidement, on peut dire que les premières "mesures 16-18 ans" se sont transformées au cours des années 80 en un ensemble d'opérations... visant les moins de 25 ans. Car au-delà, les personnes en difficultés sont censées relever du "volet insertion" du Revenu Minimum d'Insertion.

Nul ne peut en effet prétendre au RMI avant 25 ans, à l'exception des chargés de famille. En considérant qu'en-deçà, les jeunes bénéficient d'autres dispositifs (stages, formation, etc.) et surtout de la prise en charge par leurs parents, le législateur a introduit une borne chronologique de la jeunesse financièrement dépendante, sur laquelle tous les dispositifs d'insertion se sont alignés. Cette limite d'âge du RMI pose pourtant de grands problèmes à de nombreux jeunes qui se retrouvent sans ressources (Bessin, 1996). La Commission d'évaluation du RMI avait d'ailleurs souligné cette défaillance du dispositif et de nombreuses initiatives locales sous forme de "RMI jeune" émanant des Conseils Généraux tendent à la combler. Les députés ont cependant opté pour le maintien de cette frontière délimitant en quelque sorte le registre de la formation pour les uns et celui de l'assistance pour les autres : les jeunes devant échapper à ce dernier afin de leur laisser l'espoir d'accéder à un emploi. Cet argument révèle au fond les ambiguités de la flexibilité temporelle et des difficultés de la société à l'affronter.

\footnotetext{
${ }^{1}$ ) A l'initiative de Bertrand Schwartz, les opérations "Nouvelles qualifications" étaient destinées aux personnes non qualifiées, associées à l'aide de tuteurs à la modernisation des entreprises, dans une démarche d'adaptation mutuelle. Les CFI ont repris l'idée de chèque formation en donnant une "deuxième chance" aux personnes concernées.
} 


\section{La prégnance des modèles de la société salariale}

La déchronologisation dans l'application des dispositifs d'insertion correspond au processus de précarisation qui atteint l'ensemble de la population, au-delà des spécificités d'âge. Mais si certaines mesures d'insertion dépassent le public jeune, les résistances sont encore fortes. Les politiques publiques éprouvent d'énormes difficultés à dépasser des logiques sectorielles et catégorielles. Aussi, même si le risque d'exclusion menace toutes les populations confrontées à la difficulté d'entrer ou de revenir en activité, on en reste encore à définir des politiques d'insertion des jeunes et la pression de l'âge se renforce lorsqu'il s'agit d'entrer en activité. Dépasser 35 ans devient par exemple un lourd handicap pour l'accès à de nombreux postes, notamment d'encadrement. Le modèle du travail salarié stable, préparé par une période de formation initiale, formule encore largement le modèle normatif du parcours de vie dans les politiques de l'emploi. Or, les mutations temporelles, à l'origine desquelles les dérégulations du marché du travail tiennent une grande place, suggèrent une profonde reformulation des modes de régulation biographique.

\section{Quelle régulation post-fordiste du biographique?}

L'organisation de l'existence en âges auxquels on affecte un certain nombre d'attributs participe de l'intégration d'une société. L'ordre des âges contribue à l'ordre des sociétés. Ce mode de régulation fordiste du biographique (Brose, 1995) relève d'une approche fonctionnelle qui donne à chacun une place déterminée correspondant à son statut. Cette conception de la société dans son unité organique, avec ses éléments en interdépendance, est aujourd'hui mise à mal avec ce que l'on appelle "la crise du lien social". En fait, si le lien social se transforme, c'est le fondement de l'intégration dans la société qui est en crise. C'est pourtant cette intégration qui définissait justement l'adultéïté, pivot du modèle de l'institution biographique. Or, les processus de vulnérabilisation fragilisent en premier lieu le statut d'adulte, tel qu'il était défini par une intégration stable et définitive dans le travail et la famille.

\section{Enchevêtremement et désynchronisation des temps sociaux, arasement des seuils}

La réversibilité des actes et l'incertitude des statuts engendrent des parcours de vie professionnelle ou familiale qui ne peuvent plus être pensés de façon unilinéaire et irréversible. Les trajectoires biographiques devant être sans cesse réajustées, ce qui mobilise chez les individus des capacités d'adaptation toujours plus importantes pour échapper à l'exclusion, la notion d'itinéraire correspond mieux à l'incertitude qui prévaut dans les temporalités individuelles. Dans ce cadre, la conception de la formation initiale, bien distincte de son application dans la production et qui préparait à une carrière professionnelle dans un même poste pour une longue période, laisse la place à de larges recoupements entre production et formation. Plus généralement, l'évolution des rapports sociaux de sexe, avec une indépendance et une place croissantes des femmes dans la société, ne permettent plus de penser les sphères familiales et professionnelles de manière autonome. Non seulement formation et production, mais également temps familiaux et temps professionnels tendent à s'enchevêtrer. Les carrières semblent ainsi moins standardisées, pouvant allier périodes de formation, de chômage, de travail, d'éducation des enfants ou de congés sabbatiques. Cet enchevêtrement des temps sociaux relève de l'incapacité du travail à structurer l'ensemble des registres de la vie, sans pour autant qu'un autre temps dominant n'émerge. Dès lors, on assiste à une déconnexion des calendriers biographiques. Ainsi, les étapes qui scandaient le cours de vie sont beaucoup plus difficiles à percevoir. L'évolution de l'institution familiale le montre bien. Le départ des enfants du domicile parental 
n'est pas seulement beaucoup plus long à venir, il est également plus difficile à percevoir et à dater. Le mariage est devenu, pour ceux qui se marient encore aujourd'hui, l'aboutissement d'un processus continu et progressif de formation des couples, parfois après la naissance des enfants. Ces processus sont surtout de plus en plus réversibles : si l'on peut encore parler d'étapes, l'ordre de celles-ci n'est plus prévisible.

Les seuils, qui déterminaient les entrées et les sorties dans des positions stabilisées (Théry, 1993), pouvaient symboliser des temporalités biographiques empreintes du Chronos. Aujourd'hui, tout se passe comme s'il n'y avait que des accès partiels et réversibles à des statuts incertains, ce que résume bien Louis Roussel : "Les biographies traditionnelles étaient, elles, scandées par une série de ruptures : rupture par le mariage entre le célibat et les responsabilités familiales; rupture aussi entre le légitime et l'illégitime, que ce soit en terme d'union ou de filiation; ruptures dues au rang, à la parenté, aux statuts. Les seuils avaient une double fonction: dans le présent, ils rendaient une situation incompatible avec les autres; dans le temps, ils marquaient les points d'irréversibilité. Les seuils introduisaient donc celui qui le franchissait dans une situation nouvelle, distincte et en quelque sorte définitive. C'est à l'arasement progressif de ces seuils que nous assistons aujourd'hui." (Roussel, 1988, p.224)

Si les catégories politiques et administratives sont fortement interrogées par ces évolutions, celles du sociologue pour décrire les phénomènes sociaux le sont également.

\section{Nouveaux âges de la vie ou brouillage des âges?}

Ces mutations temporelles de la société impliquent une transformation des perceptions des catégories d'âge. Les travaux sur la jeunesse par exemple en attestent. Une désynchronisation des temps sociaux est apparue notamment dans les périodes de transition biographique. Il s'ensuit une déconnexion des seuils d'âge : certains droits sont acquis à des âges plus précoces alors que d'autres, comme celui du travail, s'exercent à des âges plus avancés. On a aussi évoqué la déritualisation des passages et parlé de calendriers différés. Dès lors, les analyses concluent à l'élaboration d'un "nouvel âge de la vie", "période moratoire" qui tend à s'allonger, entre "les deux âges bien définis ${ }^{1}$ que sont l'adolescence et l'âge adulte" (Galland, 1990, p.540). Cette hypothèse ne retient qu'une partie des mutations temporelles. Elle suggère seulement un déplacement des transitions, en gardant l'idée de passages successifs dans des rôles prescrits correspondant à des statuts, et ce en référence à un statut d'adulte. Mais le problème, avec la crise du lien social, l'arasement des seuils d'âge et des transitions, vient justement de la difficulté à bien définir cette figure de l'adulte, comme on pouvait le faire auparavant avec l'accès au marché du travail, à un logement indépendant et la formation d'une famille. Ces différents calendriers s'autonomisent aujourd'hui, et ces inscriptions, lorsqu'elles surviennent, ne sont plus forcément définitives. Bon nombre de personnes de quarante ans peuvent aujourd'hui se trouver à refonder une famille, à rechercher un emploi ou à réengager une formation : le "jeune père" de cinquante ans ou le "vieux chômeur" de longue durée de trente-cinq ans contribuent au brouillage des âges.

Les mêmes questions se posent pour la fin du parcours de vie. L'élévation de l'espérance de vie en bonne santé, au coeur des changements de perception des temporalités biographiques et des rapports intergénérationnels, ne permet plus d'appréhender globalement la vieillesse : retraite et grande vieillesse se sont dissociées. L'effet de construction d'un nouvel âge (le quatrième) est amplifié du fait que cette population, très hétérogène, représente un marché en pleine constitution pour nombre d'institutions et d'agents spécialisés. D'une certaine façon, les dernières périodes de la vie sont encore en cours d'institutionnalisation (Lalive d'Epinay, 1994). La légitimité dont se targuent tous ces intervenants potentiels s'appuie sur des catégories réputées "objectives", notamment celles de la médecine ou de l'épidémiologie, ce qui nourrit certains débats destinés à donner un âge au quatrième âge ("Commence-t-il à 75 ou 80 ans ?" s'interrogent-on parfois

\footnotetext{
1) C'est moi qui souligne.
} 
sérieusement !). La concurrence qui règne dans ce champ définit également les enjeux de la catégorie de "dépendance". Celle-ci mobilise d'autres critères que l'âge pour intervenir auprès des personnes âgées ${ }^{1}$, notamment parce qu'elle ne leur est pas spécifique a priori (Cf. toxicomanes, sidéens, etc.).

Quoi qu'il en soit, les catégories du sociologue pour penser les temporalités biographiques ne recouvrent pas les catégories des institutions. C'est pourquoi le renouvellement qu'impliquent les mutations temporelles de la société passe plus par une investigation approfondie sur les repères temporels du cours de vie, que par une multiplication des âges de la vie (la post-adolescence, la pré-vieillesse ou le quatrième âge) correspondant à de nouveaux champs spécialisés,. On assiste en effet à une dérégulation partielle des transitions, à l'arasement des seuils d'âge et à des dissociations des expériences biographiques qui limitent les analyses se fondant sur une institution biographique normative. Nous devons faire plus modestement le constat, si l'on appréhende l'ensemble du parcours de vie, d'une déstandardisation et d'un brouillage des âges.

Les incidences d'une certaine flexibilité sur les temporalités biographiques posent les enjeux d'une régulation qui ne s'effectue plus sur le modèle traditionnel d'une normativité référentielle, c'est-à-dire d'une intervention des institutions exerçant une normalisation des conduites en référence à un modèle unique et universel de parcours de vie (Commaille, 1994). C'est toute la difficulté des institutions à inventer d'autres critères que ceux de l'âge chronologique, qui apparaîssent encore plus rigides avec l'évolution des temporalités vécues. Plus généralement, l'instabilité et l'incertitude nous incitent à sortir d'une représentation du temps unique et linéaire du Chronos. Enjeu de taille, car n'est-ce pas rompre également avec la tradition d'une rationalité cartésienne?

\section{Références bibliographiques}

Balandier, G. (1985) Le détour, pouvoir et modernité, Paris : Fayard, 271 p.

Bessin, M. (1994) La "police des âges", entre rigidité et flexibilité temporelle, Temporalistes, 27(septembre ), 8-13 et 28(décembre), 10-15.

Bessin, M. (1996) Quelle autonomie dans la dépendance financière ? Pour un débat sur les revenus sociaux des jeunes, Agora / Débats Jeunesses, 3 (1), 7-10.

Bessin, M. (1997) Les paradigmes de la synchronisation : le cas des calendriers biographiques, Information sur les sciences sociales / Social science information, Vol.36, $\mathrm{N}^{\circ} 1$, mars.

Bourdieu, P. (1963) La société traditionnelle: attitude à l'égard du temps et conduite économique, Sociologie du Travail, janvier mars, 24-44.

Brose, HG. (1995) In search for a post-fordist life-cycle, forum "Gender et emploi du temps", Florence : Institut Universitaire Européen.

Castel, R. (1995) Les métamorphoses de la question sociale. Une chronique du salariat, Paris : Fayard, 490 p.

Chamboredon, JC. (1991) Classes scolaires, classes d'âge, classes sociales : les fonctions de scansion temporelle du système de formation, Enquête, cahiers du CERCOM, 6 (juin), 121-143.

Commaille, J. (1994) L'esprit sociologique des lois. Essai de sociologie politique du Droit, Paris: PUF, 278 p.

Conninck, F. de, Godard, F. (1990) L'approche biographique à l'épreuve de l'interprétation, Revue Française de Sociologie, XXXI, 1, 23-54.

Galland, O. (1990) Un nouvel âge de la vie, Revue française de sociologie, XXXI, n²4, 529-551.

Gaullier, X., (1988) La deuxième carrière, âges, emplois, retraites, Paris : Le Seuil, 408 p.

Grossin, W. (1996) Pour une science des temps, Toulouse : ed. Octares, 268 p.

Guillemard, AM. (1993) Emploi, protection sociale et cycle de vie : résultats d'une comparaison

${ }^{1}$ ) Ce qui n'exclut évidemment pas le caractère arbitraire de certains critères utilisés dans les diverses commissions... 
internationale des dispositifs de sortie anticipée d'activité. Sociologie du travail, n³, 257-284. Hall, ET. (1983) La danse de la vie, Paris : Le Seuil, Points anthropologie, trad. fr. 1984, 286 p. Hartog, F. (1995) Temps et histoire, "Comment écrire l'histoire de France ?", Annales, Histoire, Sciences Sociales, $n^{\circ} 6$, novembre-décembre, 1219-1236.

Lalive d'Epinay, C. (1994). La construction sociale des parcours de vie et de la vieillesse en Suisse au cours du XXème siècle, (127-150). In : Heller G. (Ed.), Le poids des ans, une histoire de la vieillesse en Suisse romande, Lausanne : SHSR \& Editions d'en bas, 180 p.

Landes, DS. (1983) L'heure qu'il est. Les horloges, la mesure du temps et la formation du monde moderne, Paris : Gallimard, trad. fr. 1987.

Neugarten, B., Hagestad, GO. (1976) Aging and the life course. In Handbooks of aging and the social sciences, Binstock R., Shanas E (ed.), New York.

Percheron, A. (1991) Police et gestion des âges, (111-139). In Percheron A., Rémond R. (dir.), Age et politique, Paris : Economica, $270 \mathrm{p}$.

Pomian, K. (1980) La crise de l'avenir, Le débat, Nº7, décembre, 5-17.

Roussel, L. (1989) La famille incertaine, Paris : O.Jacob, 284 p.

Sue, R. (1994) Temps et ordre social, Paris : PUF, 313 p.

Théry, I. (1993) L'arasement des seuils, une perspective sociologique sur le temps familial contemporain, (84-90). In : La nuit de Ville-Evrard, Temps, Mémoire, Chaos, Colloques 19901992, Paris, Descartes et Cie, 248 p.

Théry, I. (1996) Le démariage, épicentre des mutations familiales, Projet, 247 (septembre), 1726.

Thévenot, L. (1979) Une jeunesse difficile, les fonctions sociales du flou et de la rigueur dans le classement, Actes de la recherche en sciences sociales, $\mathrm{N}^{\circ} 26-27$, mars avril, 3-18.

Valabregue, C., Berger-Forestier, C., Langevin, A. (1982) Ces maternités que l'on dit tardives, Paris : Laffont, 225 p.

Zerubavel, E. (1982) La standardisation du temps : une perspective socio-historique, trad. fr. Politix, n 10-11, 2ème \& 3ème trimestre, 1990. 